\title{
Understanding Disabilities among the Elderly in China: Important Factors, Current Situation, and Future Perspective
}

\author{
Peng Tian, ${ }^{1}$ Gengyuan Wang, ${ }^{1,2}$ Yaping Liu, ${ }^{1}$ and Juan Zhou $\mathbb{D}^{3}$ \\ ${ }^{1}$ College of Public Administration, Central China Normal University, Wuhan, China \\ ${ }^{2}$ Xingyi Normal University for Nationalities, Xingyi, China \\ ${ }^{3}$ The School of Public Management, South-Central University for Nationalities, Wuhan, China \\ Correspondence should be addressed to Juan Zhou; zjzhoujuan@163.com
}

Received 20 November 2020; Revised 5 February 2021; Accepted 11 March 2021; Published 31 March 2021

Academic Editor: Maria Alessandra Ragusa

Copyright (C) 2021 Peng Tian et al. This is an open access article distributed under the Creative Commons Attribution License, which permits unrestricted use, distribution, and reproduction in any medium, provided the original work is properly cited.

The physiological functions of various organs and tissues of the elderly begin to decline with the growth of age, and the body is more and more affected by various degenerative diseases, resulting in the decline of the overall sports ability. As a result, some elderly people even become unable to live independently. This paper describes the current situation of the elderly in China by using the data of the elderly over 60 years of age in the 2015 China Health and Retirement Longitudinal Survey. In addition, Activities of Daily Living scale was used to determine the degree of disability, and Stata 13.0 software was used for multiple regression analysis, focusing on the analysis of the factors affecting the disability of the elderly. According to the research results, the paper puts forward the future trend from multiple perspectives and puts forward specific countermeasures and suggestions to alleviate the disability of the elderly or reduce its adverse effects. This will help China become an aging friendly country.

\section{Introduction}

1.1. Literature Review. At present, there is relatively little research on the elderly with disabilities in China; however, this issue has gradually received more attention from scholars. Most of the existing studies have focused on providing elderly care services in the context of population aging. Furthermore, most of them used small-sample-size questionnaire surveys to analyze the regional long-term care situation of the elderly who were disabled and its influencing factors, or to analyze the sharing of social and family responsibilities from the perspective of caregivers. In China, some studies have assessed the self-care ability of the elderly, with most of them following evaluation metrics used internationally. Examples include the Sample Survey of the Aged Population in Urban/Rural China (SSAPUR), the Chinese Longitudinal Healthy Longevity Survey (CLHLS), and the China Health and Retirement Longitudinal Survey (CHARLS). Gao pointed out that government-provided care services are more readily accepted by the elderly living in rural areas than other forms of care. In addition, this population has great potential demand for daily life care services, but it is difficult to implement these services effectively [1]. Liu argues that, in general, the current elderly care system focuses on daily life care and does not provide enough spiritual comfort. In addition, by examining relevant literature [2], Wang and Zheng found that an increase in the number of the elderly in China who are disabled is an inevitable trend, especially considering China's aging population [3]. The current imbalance in the supply and demand of care for the elderly with disabilities requires urgent attention. Many factors influence the deterioration of human bodily functions. The deterioration of the elderly people's ability to take care of themselves, to some extent, stems from the bodily deterioration that occurs over time. Exercise can reduce the chance of death and has a positive effect on reducing the impairment of self-care ability [4]. The activity of the elderly gradually decreases as they age, with females being less active than males. A decline in physical function accompanies a decrease in the frequency of activity. However, some scholars have reached different conclusions. Thus, Bauman et al. argues that people's mobility is 
significantly correlated with age and gender. Through analysis and comparison of data from several countries, it was found that young men had a higher capacity for physical activities than women. At the same time, for the elderly, there were no any significant differences in their capacity for activities between genders [5]. Zhao believes that there is a nonlinear relationship between gender, age, and health, and that gender has a significant impact on health. The age of 30 is taken as a critical indicator as it has been found that this is the point when health starts declining. However, men's health starts deteriorating at an earlier age than women's [6].

Huang pointed out that there was a clear positive correlation between nursing needs and age. In other words, the nursing needs and nursing time of the elderly are greater than those of younger people [7]. According to Yao also argued that disabilities increased the elderly people's dependence on family [8]. Zhou [9] argued that there was a strong correlation between the socioeconomic status of the elderly in urban and rural China and the loss rate of the Activities of Daily Living (ADL) scale. Moreover, Zhuang et al. found that the group aged $\geq 80$ years accounted for $93 \%$ of the elderly with disabilities and that the number of females was twice that of their male counterparts [10].

For research purposes, different scholars use different instruments to measure and gauge self-care abilities in daily life. The most widely used instruments are the Katz Activities of Daily Living (ADL) and the Lawton Instrumental Activities of Daily Living (IADL) scales [11-13]. The ADL includes the ability to dress, bath, eat, get in and out of bed, stand/squat when using the toilet, and maintain continence. The IADL includes doing chores, cooking, shopping, making phone calls, managing finances, using different modes of transportation, and doing laundry [14]. Scholars combined the ADL and IADL scales to study the self-care ability of the elderly and found that there were differences in bathing, dressing, toilet use, and manual activities between genders. People older than 80 experience more difficulty in bathing themselves compared to those younger than 80. Except for eating, all other activities included under the ADL and IADL scales will gradually decline with age. The difference between genders is mainly reflected in the difference in muscle mass between men and women [15]. For the elderly living independently, gender differences in ADL are largely influenced by cultural and environmental factors, chronic disease risk factors, and health conditions [16-18]. The cumulative effect brought about by differences in social roles is that older women are more severely impaired in their ability to care for themselves than their male counterparts. Although older women have longer life expectancy than men, they face worse health conditions later in life. In addition, studies have shown that women have higher rates of obesity, muscle weakness, and contracting chronic diseases that affect their ability to take care of themselves as compared to men [19]. Muscle strength and function are higher in men than in women of all ages. The decline in muscle strength and function is more pronounced with age in women, which is a major cause of differences between genders considering selfcare abilities.
1.2. Significance of the Study. Considering the many challenges posed by aging, how healthy aging can be achieved has become an important topic for the Chinese government and society. Under the combined effects of population aging, elderly population, and low birth rates, the burden of supporting China's working population is increasing, and the problem of caring for the disabled elderly is gradually emerging. Although the proportion of severely disabled elderly people is declining as the medical and health technology improves, it is still necessary to pay attention to the elderly who are disabled and improve their quality of life.

A serious consequence of aging is an increased prevalence of diseases, especially chronic diseases. With an increase in age, there is likewise an increase in the incidence of diseases and functional disorders. The types of diseases that the elderly contract become more complex as the population ages. There is a higher risk of physical limitations and disabilities among those with multiple chronic diseases. Furthermore, there is a strong association between chronic diseases, disabilities, and long-term care needs. The aging of the elderly population accompanies the aging of the Chinese population, and the rate of aging among this subset is faster than the rate of total population aging. The number of individuals aged $\geq 80$ years will reach 43 million in 2030 . The percentage of people aged $\geq 60$ years will increase to $12.07 \%$ in 2030 from $10.44 \%$ in 2000, and it will reach 100 million in 2050 , accounting for $22.91 \%$ of the population of those aged $\geq 60$. On October 9, 2016, the National Committee on Aging, the Ministry of Civil Affairs, and the Ministry of Finance jointly released the results of the "Fourth Sample Survey on the Living Conditions of China's Urban and Rural Older Persons," [20] showing that, in 2015, the total number of disabled and semidisabled elderly nationwide reached 40.63 million, accounting for $18.3 \%$ of the elderly population. Among them, the percentage of elderly people who are totally disabled is higher in urban areas $(6.9 \%)$ than in rural areas $(5.0 \%)$. In addition, $84.3 \%$ of disabled elderly are mildly disabled, followed by $5.1 \%$ moderately disabled and $10.6 \%$ severely disabled. The Zhongmin Social Assistance Institute under the Ministry of Civil Affairs has also released, for the first time, the "Status Report on the Loss of China's Elderly," [21] showing that approximately 500,000 elderly people are lost nationwide every year, with an average of 1,370 people lost per day. In an investigation on the causes of this trend, it was found that dementia and a lack of care were the main causes. In terms of regional distribution, most of them were the elderly left behind in rural areas. The problem of disablement among the elderly population cannot be ignored and must be addressed urgently.

Due to apparent differences in the level of socioeconomic development in various parts of the country, the overall picture of the ADL impairment among the elderly in China remains to be clarified. The elderly people's ADL is influenced by a variety of factors, including individual demographic characteristics (e.g., gender, age, way of living), personal medical conditions, socioeconomic status, lifestyle (e.g., exercise or smoking), cognitive and emotional status (e.g., depression), and even geographical location. There have also been investigations that obtained different results; 
for example, some studies have concluded that self-rated sleep status and loneliness have no significant effect on ADL. This paper attempts to use the 2015 CHARLS, conducted in 28 provinces across China, to analyze the current status of disabilities and its influencing factors in the elderly aged $\geq 60$ years. The paper uses the ADL scale to measure the self-care ability of elderly people aged $\geq 60$ years.

From the findings of the study, age and education level can predict the disability level of the elderly, while disease and marriage have a significant impact on the disability level of the elderly. Based on the data of CHARLS 2011, the proportion of the elderly who are over 60 years old will continue to increase. Continuous research on the influencing factors and current situation of the disabled elderly can continuously assess the disability status of the elderly in China, help to improve the long-term care security system in China, pay attention to prevention and healthcare, and prevent the elderly from weakening, and it can be gradually included in the scope of long-term care assessment and healthcare.

\section{Research Design}

2.1. Sources. Data used in this paper are from the CHARLS follow-up survey released in 2015. The survey was organized and implemented by the National School of Development at Peking University, with a baseline survey conducted in 2011, a follow-up survey conducted in 2013 based on the 2011 survey, and another follow-up survey conducted in 2015 based on the 2013 survey. It is currently the only large-scale household survey in China that focuses on middle-aged and elderly people, with a wide geographical representation and large sample size. This study used survey data collected in the summer of 2015 and released on November 18, 2015, targeting middle-aged adults over the age of 45 . From there, elderly people over age 60 were selected, and samples with missing key variables were removed, resulting in a total of 1,293 samples. CHARLS questionnaire design refers to international experience, including the United States Health and Retirement Survey (HRS) and the European Health, Aging and Retirement Survey (SHARE). The project adopts multistage sampling, and PPS sampling method is adopted in county and village sampling stages. CHARLS first created the electronic mapping software (CHARLS-GIS) technology, using map method to make village level sampling frame. CHARLS questionnaire includes basic personal information, family structure and financial support, health status, physical measurement, medical service utilization and medical insurance, work, retirement and pension, income, consumption, assets, and community basic information. The access response rate and data quality of CHARLS are in the forefront of similar projects in the world, and the data has been widely used and recognized in academic circles. China's elderly health and pension tracking survey project covers the most complete provinces, but the sample number of the elderly aged 60 and above is the lowest. It is necessary to control the age group to analyze the stability of disability rate.
2.2. Determining Disabilities. The ADL scale developed by Katz evaluates daily activities of elderly people. It represents the self-care ability required by an individual to maintain basic daily activities, which includes the following six activities: eating, bathing, dressing, toileting, continence, and ambulating. In this study, the ADL scale was used to determine whether the elderly were disabled, which included the six indicators of eating, dressing, getting in and out of bed, bathing, toileting, and continence. Each of these indicators comes with four levels: no difficulty, able to complete with some difficulty, needs help due to difficulty, and unable to complete. If any one of them is indicated as "needs help due to difficulty" or "unable to complete," it indicates disability; otherwise, it is not disability [22]. Activities of Daily Living are basic tasks of daily life that most people are used to doing without assistance. The ability to perform ADL is used to help determine medical status for health coverage and long-term care decisions. Assisted living facilities, inhome care providers, and nursing homes specialize in providing care and services to those who cannot perform ADL for themselves. Although the self-care ability scale decomposes the independent living ability into the ability to complete several basic daily activities, there are still some differences in the process of analyzing the specific information collected by the scale and what kind of norms to follow. What is the status of self-care ability to be judged as disability? How to define basic self-care ability (BADL) or instrumental self-care ability (IADL) in the specific operational measurement? In the clinical rehabilitation nursing work, the number of patients who were damaged was usually calculated according to the basic daily self-care activities included in Katz scale, such as bathing, dressing, going to the toilet, transferring, controlling defecation, and eating. The subjects' self-care ability was divided into seven grades, and the requirements of six items for physical fitness were different. The above order was from difficult to easy. When we use this scale to measure the disabled state of the elderly in China, we also refer to this idea, but there are some differences in the setting of specific measurement activities.

Functional independence measurement (FIM) is more detailed, accurate, and sensitive index in reflecting the level of disability or the amount of help needed. It is a powerful index to analyze and judge the rehabilitation effect. FIM not only assesses ADL dysfunction caused by motor impairment, but also evaluates the impact of cognitive impairment on daily life.

FIM includes 6 aspects, including 18 items, including 13 sports ADL and 5 cognitive ADL.

The scoring system is 7 points; that is, the highest score is 7 points, and the lowest score is 1 point. The highest total score is 126 points, and the lowest score is 18 points. The score is based on the degree of independence of patients, the need for assistive devices or assistive devices, and the amount of help given by others. When applying FIM to assessment, the assessor should first make the content and action points of each activity clear. Only by following the specific content defined by each activity can the result be objective and accurate. Pay attention to the observation of the actual operation ability of patients in the evaluation, not 
only relying on their oral statement. When evaluating the ADL of the disabled, do not evaluate what they should be able to do or what they may be able to do under certain conditions. What should be examined is the actual state.

When the patient can complete a certain activity with help, the method and amount of help should be recorded in detail. The assessment should be conducted at an appropriate time and place. Usually, occupational therapists should go to the ward to observe the patients' self-care activities such as dressing, washing, shaving, or wearing make-up when they get up in the morning, so as to show the reality. If the occupational therapy department has ADL assessment settings, it must be as close to the actual living environment as possible. In order to avoid inaccuracy due to fatigue, the assessment can be completed in several times if necessary, but it should be carried out in the same place.

The purpose of reassessment of ADL is to observe the curative effect, test the treatment method, provide the basis for timely adjustment of treatment plan, and judge the prognosis. Therefore, the time for reevaluation should be arranged at the end of a course of treatment and before discharge. New dysfunction should be evaluated at any time. For items that cannot be completed independently, the therapist should further examine the factors that affect the completion of these activities, such as joint range of motion, muscle strength, balance, coordination, and sensation. ADL level is closely related to cognitive function. Therefore, for patients with ADL disorder, cognitive and perceptual functions should be further evaluated.

2.3. Research Methodology. The survey questions and answer choices related to the respondents' basic information such as gender, age, marital status, and educational background were extracted from the basic information and family module sections of the CHARLS database. Questions and answer choices related to the respondents' mobility, selfcare, and daily activities were extracted from the health status and function sections of the database. Based on those two extractions, elderly people aged $\geq 60$ years were selected, which resulted in 1,293 subjects out of 2,378 that fit the requirements. The SPSS 24.0 was used for descriptive statistics and multifactor analysis of variance, after which Stata 13 was employed for multiple linear regression equations for multifactor analysis.

2.4. Variable Selection and Description. In this paper, the dependent variable was whether the elderly aged $\geq 60$ years were disabled, as indicated by $(Y)$ (valuation: no $=0$, yes $=1$ ). The ADL scale was used to determine whether a person was disabled. The ADL difficulty variable is a measure of the elderly people's ability to perform daily activities, which includes the six ADL of dressing, bathing, eating, waking up, toileting, and continence. The values of these variables were summed up in the questionnaire, which asked about the difficulty in performing the ADL. These specific values were of practical significance. A value of 0 was assigned to variables deemed not difficult or difficult to complete. In contrast, a value of 1 was assigned to variables deemed difficult, needing help to complete, and that cannot be completed.

The main explanatory variables in this paper were age, gender, educational background, marital status, presence of chronic illness, and presence of any disabilities. The following six variables were used as control variables for the model: (1) the age of the respondents at the time of the survey; (2) gender (assigned a value of 1 for men and 2 for women); (3) educational background (primary school and below, secondary school, and university and higher, assigned a value of 1,2 , and 3 , respectively); (4) marital status (assigned a value of 1 for married and 0 for not married); (5) whether the respondents had chronic diseases: if the respondent answers yes to one of the choices, it was considered a chronic disease and assigned a value of 1 , but if the respondent answered no to both, it was not considered a chronic disease and was assigned a value of 2; (6) whether they were disabled: if the respondent answers yes, they were considered disabled and assigned a value of 1 , but if they answer no, they were not considered disabled and were assigned a value of 0 . Since the paper only analyzed whether the explanatory variables had an effect and the direction of influence, the specific values of disability, chronic illness, and marital status had no effect on the results of this regression analysis.

\section{Empirical Findings and Analysis}

3.1. Descriptive Analysis. This paper uses the multiple linear regression method for regression analysis. Considering the significance level, setting it too high will result in too few variables entering the model, which will directly affect the model's accuracy. Therefore, the significance level $p=0.05$ was set to ensure the correctness of the model. Automatically, we set the $p$ value, which is the probability that the test statistic calculated according to the sample observation results falls into the rejection domain when the original hypothesis is true. If the $p$ value is very small, this means that the probability of this situation is very small. If this happens, according to the principle of small probability, we have a reason to reject the original hypothesis. The smaller the $p$ value is, the more sufficient the reason for rejecting the original hypothesis is. Secondly, the significance level usually has two values $(0.01$ and 0.05 ). If $p<0.01$, this means that it is a strong judgment result and the original hypothesis is rejected. If $0.01<p$ value $<0.05$, this means that the judgment result is weak and the original hypothesis condition is rejected. If $p>0.05$, the results cannot reject the original hypothesis.

3.1.1. Results of the Multivariate Analysis of Variance. According to the regression results in Table 1, the F-test value of the whole model was 3.725, and the $p$ value was 0 , which passed the test. All variables passed the significance test with a confidence level of 95\%, proving that the respective variables had a significant effect on the dependent variable. Based on the regression results in Table 2 , the whole model passed the test with an F-test value of 3.625 and a $p$ value of 0 . All variables passed the significance test with a confidence level of $95 \%$, proving that the respective variables had a significant effect on the dependent variable. 
TABLE 1: Results from the multifactor analysis of variance.

\begin{tabular}{|c|c|c|c|c|c|c|}
\hline \multicolumn{7}{|l|}{ Dependent variable: disabled or not } \\
\hline Variable & $\begin{array}{c}\text { Sum of squares of } \\
\text { deviation from mean }\end{array}$ & $\begin{array}{l}\text { Degree of } \\
\text { freedom }\end{array}$ & $\begin{array}{l}\text { Mean-square } \\
\text { value }\end{array}$ & $F$ & $\begin{array}{l}\text { Statistical } \\
\text { significance }\end{array}$ & $\begin{array}{c}\text { Partial } \\
\text { eta-squared }\end{array}$ \\
\hline Correction model & $14.651 \mathrm{a}$ & 68 & 0.215 & 3.725 & 0.000 & 0.171 \\
\hline Age & 2.320 & 35 & 0.066 & 1.146 & 0.258 & 0.032 \\
\hline Educational background & 0.648 & 8 & 0.081 & 1.399 & 0.192 & 0.009 \\
\hline Marital status & 0.052 & 5 & 0.010 & 0.179 & 0.970 & 0.001 \\
\hline Gender & 0.201 & 1 & 0.201 & 3.468 & 0.063 & 0.003 \\
\hline Physical disability & 1.442 & 1 & 1.442 & 24.929 & 0.000 & 0.020 \\
\hline Brain damage/intellectual disability & 0.306 & 1 & 0.306 & 5.293 & 0.022 & 0.004 \\
\hline Blindness or partial blindness & 0.254 & 1 & 0.254 & 4.395 & 0.036 & 0.004 \\
\hline Deafness or partial deafness & 0.326 & 1 & 0.326 & 5.637 & 0.018 & 0.005 \\
\hline Stuttering or severe stuttering & 0.007 & 1 & 0.007 & 0.114 & 0.736 & 0.000 \\
\hline Hypertension & 0.029 & 1 & 0.029 & 0.509 & 0.476 & 0.000 \\
\hline Dyslipidemia & 0.056 & 1 & 0.056 & 0.963 & 0.327 & 0.001 \\
\hline Diabetes & 0.169 & 1 & 0.169 & 2.930 & 0.087 & 0.002 \\
\hline Cancer & 0.122 & 1 & 0.122 & 2.111 & 0.147 & 0.002 \\
\hline Chronic lung disease & 0.051 & 1 & 0.051 & 0.883 & 0.348 & 0.001 \\
\hline Liver diseases & 0.083 & 1 & 0.083 & 1.431 & 0.232 & 0.001 \\
\hline Heart disease & 0.197 & 1 & 0.197 & 3.412 & 0.065 & 0.003 \\
\hline Stroke & 1.188 & 1 & 1.188 & 20.537 & 0.000 & 0.017 \\
\hline Kidney disease & 0.003 & 1 & 0.003 & 0.049 & 0.825 & 0.000 \\
\hline Stomach or digestive system disease & 0.116 & 1 & 0.116 & 2.001 & 0.157 & 0.002 \\
\hline Emotional and mental problems & 0.010 & 1 & 0.010 & 0.172 & 0.679 & 0.000 \\
\hline Memory-related disorders & 0.781 & 1 & 0.781 & 13.500 & 0.000 & 0.011 \\
\hline Arthritis or rheumatism & 0.233 & 1 & 0.233 & 4.019 & 0.045 & 0.003 \\
\hline Asthma & 0.532 & 1 & 0.532 & 9.201 & 0.002 & 0.007 \\
\hline Errors & 70.803 & 1224 & 0.058 & & & \\
\hline Aggregate & 92.000 & 1293 & & & & \\
\hline Revised total & 85.454 & 1292 & & & & \\
\hline
\end{tabular}

3.1.2. Results of the Multiple Regression Analysis. The data for the variables previously described were entered into Stata 13.0, and a multiple linear regression analysis was performed. The results are shown in Table 3.

By observing the regression results from Table 3 , it is evident that the $\mathrm{F}$ value of the model is $F(6,1323)=14.64$. Prob $>F=0.000$ indicates that the model, as a whole, is very significant. In these regression results, age, marital status, presence of disability-related issues, and presence of chronic diseases are highly significant at the $95 \%$ significance level. In contrast, educational background and being retired were not significant. The univariate analysis showed that there were statistically significant differences in mobility scores for gender, educational background, marital status, children's income level, chronic disease status, and alcohol consumption.

3.1.3. Stepwise Multiple Regression. According to the regression results in Table 4, both $\mathrm{F}$ and $p$ values of the model indicate that the model, as a whole, is statistically significant. The $p$ value for all variables is $<0.05$ at the $95 \%$ significance level, indicating that all variables are statistically significant. An expansion of the variables as to whether there were disability problems and chronic diseases is shown in Table 5. Thus, among disability problems, physical disability, brain damage/intellectual disability, blindness and partial blindness, and deafness/partial deafness have a significant impact on the disabilities of the elderly. Among chronic diseases, having a stroke, memory-related diseases, arthritis/rheumatism, and asthma had a significant impact on the disabilities of the elderly.

3.2. Analysis of the Multiple Regression Model Results. There are significant differences in the inference results of disability rates from different source databases. In this paper, the disability rate was $9.25 \%$, which is significantly different from the study results by Zhang and Wei (10.48\% 13.31\%) and Yang et al. (8.18\%). Zhang and Wei's research makes full use of the completed survey data; the CLHLS, SSAPUR, and CHARLS special survey data of the same period are combined in the assessment of the disability level of the elderly, so as to overcome the size of the sample population to the greatest extent and the problem of low modulus. Although this method can increase the sample size of data and improve the accuracy of research, it will also make the structural differences between databases become a problem. We think that the comparison of continuous data based on CHARLS is also an important perspective, so it is valuable to make a comparison with the failure rate of $11.25 \%$ based on CHARLS analysis. From the intuitive data, with the improvement of medical level and personal quality, the disability rate of the same age dimension has a downward trend. This may be related to the sample composition of the data, the survey timing, and the assessment of disabilities [23, 24]. 
TABLE 2: Results from the multifactor analysis of variance.

\begin{tabular}{|c|c|c|c|c|c|}
\hline Dependent variable: disabled or not & & & & & \\
\hline Variable & $\begin{array}{l}\text { Sum of squares of deviation } \\
\text { from mean }\end{array}$ & Degree of freedom & $\begin{array}{l}\text { Mean-square } \\
\text { value }\end{array}$ & $F$ & $\begin{array}{c}\text { Statistical } \\
\text { significance }\end{array}$ \\
\hline Correction model & $14.692 \mathrm{a}$ & 70 & 0.210 & 3.625 & 0.000 \\
\hline Age & 2.329 & 35 & 0.067 & 1.149 & 0.254 \\
\hline Gender & 0.645 & 8 & 0.081 & 1.393 & 0.195 \\
\hline Educational background & 0.053 & 5 & 0.011 & 0.183 & 0.969 \\
\hline Marital status & 0.202 & 1 & 0.202 & 3.494 & 0.062 \\
\hline Presence of disability-related issues & 0.021 & 1 & 0.021 & 0.367 & 0.545 \\
\hline Suffering from chronic illnesses & 0.018 & 1 & 0.018 & 0.314 & 0.575 \\
\hline Errors & 70.762 & 1222 & 0.058 & & \\
\hline Aggregate & 92.000 & 1293 & & & \\
\hline Revised total & 85.454 & 1292 & & & \\
\hline
\end{tabular}

TABLE 3: Results of the multiple regression analysis on being disabled or not.

\begin{tabular}{|c|c|c|c|c|c|c|}
\hline Disabled or not & Coefficient & Standard deviation & $t$ & $p>|t|$ & \multicolumn{2}{|c|}{ (95\% confidence interval) } \\
\hline Age & 0.0023967 & 0.0010061 & 2.38 & 0.017 & 0.0004229 & 0.0043706 \\
\hline Gender & 0.0052931 & 0.0044926 & 1.16 & 0.248 & -0.00362 & 0.0140063 \\
\hline Educational background & 0.0002336 & 0.00429929 & 0.06 & 0.957 & -0.008188 & 0.0086553 \\
\hline Marital status & 0.313562 & 0.148443 & 2.11 & 0.035 & 0.0022352 & 0.604772 \\
\hline Presence of disability-related issues & -0.1244832 & -0.168741 & -7.38 & 0.0001 & -0.1575861 & -0.913803 \\
\hline Suffering from chronic illnesses & -0.617708 & 0.0161676 & -3.82 & 0.0001 & -0.934878 & -0.300539 \\
\hline Cons & 0.1476852 & 0.843754 & 1.76 & 0.080 & -0.017839 & 0.3132093 \\
\hline \multicolumn{7}{|l|}{$F(6,1323)=14.64$} \\
\hline \multicolumn{7}{|l|}{ Prob $>F=0.000$} \\
\hline \multicolumn{7}{|l|}{$R$-squared $=0.0623$} \\
\hline \multicolumn{7}{|l|}{ Adj $R$-squared $=0.580$} \\
\hline Root $\mathrm{MSE}=0.25366$ & & & & & & \\
\hline
\end{tabular}

TABLE 4: Results of the stepwise multiple regression.

\begin{tabular}{|c|c|c|c|c|c|c|}
\hline Disabled or not & Coefficient & Standard deviation & $t$ & $p>|t|$ & \multicolumn{2}{|c|}{ (95\% confidence interval) } \\
\hline Age & 0.0023967 & 0.0010061 & 2.38 & 0.017 & 0.0003938 & 0.3644116 \\
\hline Marital status & 0.313562 & 0.148443 & 2.11 & 0.035 & 0.0022352 & 0.604772 \\
\hline Presence of disability issues & -0.1244832 & -0.168741 & -7.38 & 0.0001 & -0.1575861 & -0.913803 \\
\hline Chronic illnesses & -0.617708 & 0.0161676 & -3.82 & 0.0001 & -0.934878 & -0.300539 \\
\hline Cons & 0.1476852 & 0.843754 & 2.79 & 0.005 & 0.0637036 & 0.3644116 \\
\hline \multicolumn{7}{|l|}{$F(6,1323)=27.64$} \\
\hline \multicolumn{7}{|l|}{ Prob $>F=0.000$} \\
\hline \multicolumn{7}{|l|}{$R$-squared $=0.0589$} \\
\hline \multicolumn{7}{|l|}{ Adj $R$-squared $=0.567$} \\
\hline Root MSE $=0.25383$ & & & & & & \\
\hline
\end{tabular}

Both age and educational background have clear impacts on the ADL, with many studies showing that health differs between genders such that women are often at a disadvantage. However, when other factors are considered, gender differences in ADL are not significant. Gender may be strongly correlated with other factors, such as age (longer life expectancy for women) and educational background (lower educational attainment for women). There are significant age differences in ADL as well. Zhang and Wei evaluated the level of disabilities in China's urban and rural elderly population using data from three Chinese surveys on the elderly (CLHLS, CHARLS, and SSAPUR). The results showed that the disability rate in China's urban and rural elderly population fluctuates due to age, gender, and urbanrural distribution of the samples [23], which is consistent with previous findings. As age increases, the prevalence of chronic disease increases, physical function decreases, and ADL levels decline among older adults. With regard to the impact of educational background, the study leans toward the conclusion that higher educational attainment leads to less ADL impairment. Older adults who are better educated tend to have better work environments, economic status, 
TABLE 5: Multiple regression results for the individual variables of the disabled.

\begin{tabular}{|c|c|c|c|c|c|c|}
\hline Disabled & Coefficient & Standard deviation & $\mathrm{t}$ & $p>|t|$ & \multicolumn{2}{|c|}{ (95\% confidence interval) } \\
\hline Age & 0.0023769 & 0.0009777 & 2.43 & 0.015 & 0.0004577 & 0.004294 \\
\hline Educational background & 0.0002456 & 0.0043722 & 0.56 & 0.574 & -0.0061215 & 0.0110335 \\
\hline Marital status & 0.0002278 & 0.0041569 & 0.05 & 0.956 & -0.0079275 & 0.008383 \\
\hline Gender & 0.0248379 & 0.0143658 & 1.73 & 0.084 & -0.0033454 & 0.0530211 \\
\hline Physical disability & -0.2177461 & 0.0413576 & -5.26 & 0.0001 & -0.2988828 & -0.1366093 \\
\hline Brain damage/intellectual disability & -0.105346 & 0.0438723 . & -2.40 & 0.016 & -0.1914162 & -0.0192759 \\
\hline Blindness or partial blindness & -0.0579315 & 0.0279862 & -2.07 & 0.039 & -0.1128358 & -0.0030272 \\
\hline Deafness or partial deafness & -0.0475304 & 0.0204548 & -2.32 & 0.020 & -0.0876594 & -0.0074014 \\
\hline Stuttering or severe stuttering & -0.0636656 & 0.105319 & -0.60 & 0.546 & -0.2702841 & 0.1429529 \\
\hline Hypertension & -0.0130158 & 0.0145785 & -0.89 & 0.372 & -0.0416165 & 0.0155849 \\
\hline Dyslipidemia & 0.0220961 & 0.0236721 & 0.93 & 0.351 & -0.0243446 & 0.0685368 \\
\hline Diabetes & -0.0422556 & 0.0260122 & -1.62 & 0.105 & -0.0932872 & 0.0087759 \\
\hline Cancer & -0.0752628 & 0.0533932 & -1.41 & 0.159 & -0.1800114 & 0.0294857 \\
\hline Chronic lung disease & -0.0229355 & 0.022411 & -1.02 & 0.306 & -0.0669022 & 0.0210313 \\
\hline Liver diseases & 0.0235449 & 0.0295659 & 0.80 & 0.426 & -0.0344586 & 0.0815483 \\
\hline Heart disease & -0.0279454 & 0.0194356 & -1.44 & 0.151 & -0.0660749 & 0.0101841 \\
\hline Stroke & -0.1752278 & 0.0372891 & -4.70 & 0.0001 & -0.2483829 & -0.1020728 \\
\hline Kidney disease & -0.0080584 & 0.0289459 & -0.28 & 0.781 & -0.0648455 & 0.0487287 \\
\hline Stomach or digestive system disease & -0.0204425 & 0.0164599 & -1.24 & 0.214 & -0.0527342 & 0.0118491 \\
\hline Emotional and mental problems & 0.0191352 & 0.061465 & 0.31 & 0.756 & -0.101449 & 0.1397194 \\
\hline Memory-related disorders & -0.2007915 & 0.0563144 & -3.57 & 0.0001 & -0.311271 & -0.0903119 \\
\hline Arthritis or rheumatism & -0.0312724 & -0.014733 & -2.12 & 0.034 & -0.0601762 & -0.0023687 \\
\hline Asthma & -0.1116816 & 0.0351583 & -3.18 & 0.002 & -0.1806563 & -0.0427069 \\
\hline cons & 2.112104 & 0.3026871 & 6.98 & 0.0001 & 1.518282 & 2.705926 \\
\hline
\end{tabular}

lifestyle habits, cognitive functions, and therefore lower rates of disability impairments.

Many factors affect disabilities among the elderly. Studies have found that diseases (especially chronic diseases) are key factors behind disabilities among the elderly. The number and types of diseases that affect the elderly show a tendency to increase gradually with age. These diseases affect the ability to see, hear, think, and react [25]. Marital status is also a key factor behind disabilities among the elderly. Generally speaking, elderly people who are unmarried, widowed, and divorced are more likely to experience negative emotions such as loneliness, repression, and depression, all of which will invariably pose a hidden danger to their physical health and will affect their daily self-care ability [26]. A study by Yang et al. [24] found that chronic disease is an important factor affecting disability. Older adults with chronic diseases have a greater risk of being disable 1.43 times greater than those without chronic diseases. Those with three or more chronic diseases have a 2.47 times greater risk than those without. The disability rate among the elderly increases significantly with age. Risk factors, such as chronic illnesses and mental health problems, also influence the continued ability of older adults to care for themselves. This finding is consistent with the results of this study.

\section{Discussion and Conclusion}

This study found that the disability rate among the elderly increases significantly with age. Risk factors such as having chronic diseases and disability-related problems also affect the continued ability of the elderly to take care of themselves. This suggests that the state should continue to strengthen prevention and control measures to address the factors affecting the disablement of the elderly, such as reducing the prevalence of chronic diseases, strengthening mental health education for the elderly, and raising awareness of selfhealth management among the elderly to improve their selfcare ability and control the disability rate. Furthermore, as the population's median age and the number of disabled elderly people continue to increase, the demand for longterm care for the elderly will continue to increase. However, presently in China, care for the elderly who are disabled mainly comes from their family as there is a relative shortage of welfare resources for their long-term care along with a slow development of social care. Therefore, the government should establish a comprehensive long-term care service system and improve the quality of long-term care services for the elderly.

Emphasis should be placed on the physical and mental health of the disadvantaged elderly, and they should be supported by both their family and society. Families and society should pay closer attention to the physical and mental health of the disadvantaged elderly people, such as those who are female, old, or disabled. For disadvantaged elderly people, their traditional attitudes still favor home or community-based care, so family members, especially their children, should take responsibility for the daily care of disadvantaged elderly people, especially those who are ill or disabled. Children of the disadvantaged elderly should try to live with them, or close to them, to provide daily care and spiritual companionship. The government should also provide professional services for the elderly in their homes, establish even more standardized and professional institutions, and provide social groups for spiritual comfort. All 
these could help to reduce the suffering that the disadvantaged elderly experience from physical illness or pain and could promptly resolve their negative psychological anxiety and depression so that they can lead a more positive and healthy life in their old age.

The massive number of disabled elderly people in China has put tremendous pressure on the country's elderly service system in addressing the challenges posed by aging. The country must recognize that healthy aging is the key to alleviating the pressures of an aging population. The insidious onset, long duration, and persistence of chronic diseases must be addressed; there is a need to innovate the prevention and control functions of the health service system for chronic diseases. The incidence of chronic diseases can be effectively reduced by disseminating information on health and healthcare, developing preventive health programs, and investing resources in maternal and child healthcare. Additionally, physical exercise and regular medical check-ups are important ways to reduce the risk of disabilities from diseases, while also maintaining health capital. Optimization of rural sports infrastructure and arrangements for regular medical check-ups for the elderly can promote the maintenance of their health capital.

Strengthening health management for the elderly and focusing on the prevention and control of chronic diseases are necessary. Chronic diseases are a major factor in the lives and health of the elderly in China, causing physical pain, inhibiting mobility and self-care abilities, and seriously affecting every-day activities. Moreover, the financial pressure and mental burden caused by chronic pain and medication make them vulnerable to anxiety, depression, despair, and other psychological problems, which further aggravate their illnesses, negatively impacting their health. Departments related to the physical and mental health of the elderly should conduct regular quality-of-life measurements of both healthy and sick elderly people. They should also conduct a comprehensive analysis based on the data derived from the health examinations so they could provide data basis and scientific guidance for community health services, thereby reducing the negative impact of chronic diseases on the quality of life. The most effective way to reduce the damage of chronic diseases is to carry out health literacy education. Only through long-term and persistent education can the elderly people's health awareness and sensitivity to chronic disease symptoms be improved. They can gradually develop positive daily healthcare awareness and behavioral habits. In carrying out health literacy education, the concept that must be adhered to is "teaching considering the individual and teaching considering the disease"; different health conditions stem from individual characteristics of the elderly. Different methods of teaching and awareness raising must be adopted for those with different health conditions, occupations, and educational background.

4.1. Theoretical Contribution. The first theoretical contribution is using new data to verify the research conclusions of Zhang and Wei, and Yang et al. on disability of the elderly in
China and discussing the possibility of different research results $[23,24]$.

The second theoretical contribution is concluding that gender has no significant effect on ADL of the elderly in China. It corrects the conclusion of many studies that gender health is unfair.

The third contribution is to verify the predictability of age and education level to ADL level through new data. The prevalence of chronic diseases in the elderly is increased, the body function is decreased, and the ADL level of the elderly will be decreased. Regarding the influence of education level, the research is close to the conclusion of "higher education level, lower ADL damage." The elderly with higher education often have better working environment, economic status, living habits, and cognitive function, so the disability rate is lower.

The fourth theoretical contribution is to reverify that disease, marriage, and other factors have a significant impact on disability of the elderly in China.

4.2. Practical Contribution and Policy Suggestions. With the aging of the population and the change of family structure, the care of the disabled elderly has gradually evolved from family responsibility to the whole society. It is necessary to take appropriate preventive measures and public policies to deal with it. Based on the concept of "active aging" and "healthy aging," this study aims to maintain and enhance the feasible ability and function of the elderly through the improvement of care resources and care environment for the elderly in China, improve their social participation opportunities and subjective well-being, and help build an aging friendly country and sustainable society.

The first practical contribution: The research on the situation and affecting factors of the disabled elderly in China is conducive to the construction of the evaluation system for the disabled elderly. By learning from the international long-term care needs assessment indicators, a composite evaluation index system is established to comprehensively assess the physical and mental functions, care resources, social environment, and personal will of the disabled elderly, so as to enhance their feasible ability and function. The assessment system of the disabled elderly is the basis of long-term care insurance system. Combined with the theory of feasible ability and international experience, the assessment index of care needs (disability assessment index) should include physical structure, ADL, IADL, cognitive and behavioral problems, action ability, family care resources, social participation ability, and psychological status. However, the current care needs assessment in China's pilot areas fails to reflect the needs of the cognitive disabled. With the continuous expansion of pilot areas in China, the scientific assessment tools for long-term care rating have been promoted. Firstly, based on the concept of maintaining and restoring the feasible ability of disabled elderly people, a composite disability assessment scale was established. Secondly, using the method of dividing the degree of disability by care time in advanced countries for reference, the research on care time of disabled care 
projects was gradually carried out, and the evaluation information of China was collected. The objective is to evaluate the consumption of nursing resources and to classify the disability level more scientifically. The establishment of needs assessment level and care level matching, through the scientific and objective division of disability level and corresponding care level service, is conducive to the rational allocation of long-term care resources for the disabled elderly in China. In order to better estimate the number of potential long-term care needs and the demand for care services and manpower and to plan the care level, payment standard, and human resource development strategy for long-term care insurance, which is limited by the existing database, it is suggested that the responsible units of long-term care insurance should design relevant questionnaires according to the multidimensional evaluation system and conduct national long-term care demand surveys regularly, which can be used as a reference for longterm care policy planning.

The second practical contribution: The research on the situation and affecting factors of the disabled elderly in China is conducive to improving the long-term care security system and establishing the care management system. Referring to the care management system established in developed countries and regions, after the assessment of disability level by assessors, the care management specialist, in combination with the care level, formulates the most appropriate care plan according to the multiple needs of the caregivers within the scope of insurance benefits, integrates and connects the care resources, and regularly modifies them according to the changes of individual needs, so as to effectively build the disabled persons and long-term care resources bridge. As the "gatekeeper" of the long-term care insurance fund, in order to maintain the public welfare and fairness, the long-term care management department, a subsidiary of the health and health management committee, should be responsible for the assessment and care plan arrangement. The care administrators should cooperate closely with community service resources (designated institutions) and informal care resources (family members) to integrate the resources of formal care and informal care, respect the choice of the elderly, and link and coordinate the arrangement of long-term care services to respond to their care needs.

The current long-term care insurance system in China has not yet set up a care management link. In actual operation, some pilot areas, such as Guangzhou, provide care service packages, which are selected by the personnel who have passed the disability assessment; or those who choose to provide for the aged who are cared by institutions, the care managers will assess the disability status and draw up care plans.Although the former retains the autonomy of the caregivers, the information asymmetry exists in the demander, which may not match the supply and demand scientifically; in the latter, the institutional staff are often considered by the business performance and overestimate the level of the need for care, so as to obtain higher insurance benefits. The construction of long-term care system needs not only care insurance and service institutions, but also a sound care management system and human resources system.

The third practical contribution: The research on the situation and affecting factors of the disabled elderly in China is conducive to the national level to pay attention to the prevention and treatment of elderly weakness and gradually bring them into the scope of long-term radiation assessment and healthcare. In Japan, we attach great importance to the need to support level 1 and level 2 prevention and care work. In particular, $3 \%$ of the nursing insurance premium is allocated to the high-risk elderly for preventive care services. At the same time, Taiwan has also added the elderly frailty assessment (SOF) in the disability assessment scale to identify the high-risk disabled elderly, and the relevant expenses are included in the long-term care fund payment scope. Therefore, this paper suggests that, at the initial stage of long-term care insurance in China, we should not only focus on the care needs of the elderly with moderate or severe disability, but also include preventive healthcare measures, such as setting up community-based care stations to provide health promotion and health examination for the elderly and encourage social participation of the elderly, so as to prevent the occurrence of and deepen the degree of disability of the elderly.

The fourth practical contribution: The research on the situation and affecting factors of disabled elderly in China is conducive to strengthening the support of caregivers and improving their skills.

In the assessment of care needs, it is necessary to assess the degree of physical dependence and family care ability and willingness and further assess the demand for formal services. The assessment of long-term care needs in many countries and regions covers the assessment of the care burden and ability of family caregivers. Family caregivers are included in the target of long-term care services, and corresponding support is provided for family caregivers in longterm care service projects, including the provision of care resources, long-term care knowledge and skills training, breathing service, family care service, and community volunteers. We should build special consultation service line, interactive network platform, volunteer service platform, and emergency placement system to provide more care and support for family caregivers from both software and hardware levels.

\section{Data Availability}

The data of the study can be obtained after the website is registered, http://charls.pku.edu.cn/.

\section{Conflicts of Interest}

The authors declare that they have no conflicts of interest.

\section{Acknowledgments}

This project was funded by (1) the Fundamental Research Funds for the Central Universities (South-Central University for Nationalities: CSY19018), "Study on the Basic 
Security of Long Term Care from the Perspective of Intergenerational Justice," and (2) the Theoretical Innovation Project of Guizhou Province, project name: "Study on the Integrated Development Strategy of Education and Urbanization in Guizhou Province," under project number: GZLCLH-2018-121.

\section{References}

[1] J. Gao, "Research on the demand of daily life care services for the elderly in rural areas," Master and doctoral dissertation, Library of Shandong University, Jinan, China, 2010.

[2] X. Liu, "On the particularity of rural elderly care in China," Theorists, vol. 1, pp. 230-231, 2006.

[3] L. Wang and S. Zeng, "Research review on the status of disabled elderly and elderly long term care insurance," Journal of Population, vol. 06, pp. 86-91, 2015.

[4] P. A. Latorre-Roma'n, J. A. Laredo-Aguilera, F. GarcíaPinillos, V. M. Soto-Hermoso, and J. M. Carmona-Torre, "Physical activity, weight and functional limitations in elderly Spanish people:the National Health Survey (2009-2014)," The European Journal of Public Health, vol. 28, no. 4, pp. 778-783, 2018.

[5] A. Bauman, F. Bull, T. Chey et al., "The international prevalence study on physical activity: results from 20 countries," International Journal of Behavioral Nutrition and Physical Activity, vol. 6, no. 1, pp. 21-61, 2009.

[6] Z. Zhao, "Health status of China's rural population and factors influencing it," Managing the World, vol. 3, pp. 78-85, 2006.

[7] C. Huang, "Long term care needs of the elderly in Beijing," Health Economics Research, vol. 04, pp. 28-30, 2005.

[8] Y. Yao, "The influence of elderly disability on the changes of family pension function in China," Population Study, vol. 33, no. 2, pp. 58-68, 2009.

[9] L. Zhou, "The relationship between the social and economic status of the elderly and the loss of self-care ability in daily life," Population and Development, vol. 18, no. 3, pp. 83-86, 2012.

[10] X. Zhuang and L. Zhang, "Analysis of the elderly disabled elderly,” Population Journal, vol. 38, no. 3, pp. 47-57, 2016.

[11] I. Vergara, A. Bilbao, M. Orive, S. Garcia-Gutierrez, G. Navarro, and J. Quintana, "Validation of the Spanish version of the Lawton IADL Scale for its application in elderly people," Health and Quality of Life Outcomes, vol. 10, no. 1, p. 130, 2012.

[12] D. D. Dunlop, L. M. Manheim, M.-W. Sohn, X. Liu, and R. W. Chang, "Incidence of functional limitation in older adults: the impact of gender, race, and chronic conditions," Archives of Physical Medicine and Rehabilitation, vol. 83, no. 7, pp. 964-971, 2002.

[13] S. Koukouli, I. G. Vlachonikoils, and A. Philalithis, "Sociodemographic factors and self-reported functional status; the significance of social support," BMC Health Services Research, vol. 2, no. 20, 2002.

[14] I. E. Mattos, C. N. do Carmo, L. M. Santiago, L. L. Luz et al., "Factors associated with functional incapacity in elders living in long stay institutions in Brazil:a cross-sectional study," BMC Geriatrics, vol. 14, no. 47, 2014.

[15] S. Demura, S. Sato, M. Minami, and K. Kasuga, "Gender and age differences in basic ADL ability on the elderly:comparison between the independent and the dependent elderly," Journal of Physiological Anthropology and Applied Human Science, vol. 22, no. 1, 2002.
[16] U. Soon, "Asberg KH Assessment of activities of daily living in the elderly: a study of a population of 76-year-olds in Gothenberg, Sweden," Scand Journal of Rehabilitation Medicine, vol. 23, pp. 193-202, 1991.

[17] V. L. Lamb, "Gender differences in correlates of disablement among the elderly in Egypt," Social Science \& Medicine, vol. 45, no. 1, pp. 127-136, 1997.

[18] K. A. Ranberg, K. Christensen, B. Jeune, A. Skytthe, L. Vasegaard, and J. W. Vaupel, "Declining physical abilities with age:a cross-sectional study of older twins and centenarians in Denmark," Age and Aging, vol. 28, pp. 378-383, 1999.

[19] L. A. Tseng, M. J. Delmonico, M. Visser et al., "Body composition explains sex differential in physical performance among older adults," The Journals of Gerontology Series A: Biological Sciences and Medical Sciences, vol. 69, no. 1, pp. 93-100, 2014.

[20] Ministry of Civil Affairs Portal, "Department $\mathrm{H}$ released the results of the fourth China city sampling survey on the living conditions of many elderly people [EB/OL]," 2016, http://www. mca.gov.cn/article/zwgk/mzyw/201610/20161000001974.shtrnl.

[21] CCTV news website, 2016, http://news.cctv.con/2016/10/ 09ARTIUQhpkvofzhG4okAOzeXe161009.shtml.

[22] Y. Wang, Y. Feng, W. Hou et al., "Investigation of long-term care needs of disabled elderly and home caregivers of Han Chinese in Xinjiang," China Family Medicine, vol. 16, no. 14, pp. 1268-1273, 2013.

[23] W. Zhang and M. Wei, "How high is the level of disablement among the elderly in China? a comparison of multiple data sources," Demographic Research, vol. 39, no. 3, pp. 34-47, 2015.

[24] F. Yang, X. Hao, T. Bo et al., "Analysis of the current state of disability in China's elderly and its influencing factors. based on CHARLS Empirical analysis of the data," Health Economics Research, vol. 11, pp. 7-10, 2016.

[25] K. Takeda, MizusimS, O. Tochikubo et al., "Relationship between activities of daily living and rresidential environment for patients receiving home oxygen therapy," Japanese Journal of Public Health, vol. 49, no. 7, pp. 683-693, 2002.

[26] J. M. Perkins, H. Y. Lee, K. S. James et al., "Marital status, widowhood duration, gender and health outcomes:A crosssectional study among older adults in India," BMC Public Health, vol. 16, no. 1, p. 1032, 2016. 